\title{
Biological effects of fibrous and particulate substances and related areas: foreword
}

\author{
Reviews of the symposium "Biological Effects of Fibrous and Particulate Substances \\ and Related Areas" as organized by the Study Group on Fibrous and Particulate \\ Substances (SGFPS) of the 78th Annual Meeting of the Japanese Society \\ for Hygiene (JSH), 2008
}

\author{
Takemi Otsuki \\ Published online: 26 May 2009 \\ (C) The Japanese Society for Hygiene 2008
}

Among the symposia organized by the various study groups of the Japanese Society for Hygiene (JSH), the Study Group on Fibrous and Particulate Substances (SGFPS) presented the symposium "Biological Effects of Fibrous and Particulate Substances and Related Areas", chaired by Dr Seishiro Hirano (Environmental Health Sciences Division, National Institute for Environmental Studies, Tsukuba, Japan) and Professor Takemi Otsuki (Kawasaki Medical School, Japan), on 29 March, 2008 as part of the program of the 78th Annual Meeting of the Japanese Society for Hygiene (JSH).

The SGFPS started its activity in 2006. Medical and social issues regarding asbestos-related malignant pleural mesothelioma (MPM) were raised previously in the summer of 2005. This issue has made people in Japan aware that asbestos causes malignant diseases, for example MPM, lung cancers, and asbestosis, through both industrial and environmental exposure. At the 76th Annual Meeting of the JSH held in 2006 at Ube, Yamaguchi, Japan, the SGFPS held a symposium entitled "Asbestos: Science and Society." The SGFPS then had two special sessions at the 78th Annual Meeting of the JSH. One session involved a symposium entitled "Malignant Mesothelioma", for which our study group invited mesothelioma specialists in Japan to improve recognition of this disease and develop preventive measures for this disease in the field of hygiene. The speakers were Professor Kouki Inai from Hiroshima University, Dr Yoshitaka Sekido from Aichi Cancer Center Research Institute, Professor Okio Hino from Juntendo University School of Medicine,

T. Otsuki $(\square)$

Department of Hygiene, Kawasaki Medical School,

577 Matsushima, Kurashiki 7010192, Japan

e-mail: takemi@med.kawasaki-m.ac.jp and Professor Takashi Nakano from Hyogo College of Medicine. Reviews of speaker presentations were published in Vol. 13, No 2 of this journal as a special feature on malignant mesothelioma. The other session was a workshop, chaired by Professor Otsuki, in which five members of the SGFPS presented their research topics. Dr Ayako Takata (St Marianna University School of Medicine, Japan) presented "Hazard assessment of product materials of the innocuous process of asbestos" and Dr Yasumitsu Nishimura (Kawasaki Medical School) focused on the "Functional alteration of the NK cells upon the long-term exposure to asbestos". In addition, Dr Akiyo Tanaka and Miyuki Hirata (Kyushu University, Graduate School of Medicine, Japan) lectured on "Pulmonary toxicity of dispersed carbon nanofiber instilled intratracheally in rats" and Dr Seishiro Hirano delivered a presentation on the "Cellular uptake and translocation of nanoparticles". Furthermore, Professor Shuichi Adachi (Sagami Women's University, Japan) made an additional presentation focusing on the "Prevention of mesothelioma by anti-oxidants". Many people attended the symposium and workshop, and members of the JSH were encouraged to investigate the prevention and treatment of asbestos-related MPM.

During the 78th Annual Meeting of the JSH, the SGFPS held a special symposium entitled "Biological Effects of Fibrous and Particulate Substances and Related Areas" chaired by Dr Hirano and Professor Otsuki. The purpose of this symposium was to recognize recent developments related to fibrous and particulate substances. The four members of the SGFPS introduced their research highlights that improved our understanding of recent scientific advances regarding fibrous and particulate substances. Dr Hirano summarized recent topics concerning nanoparticle research as "Worldwide trend for a safety evaluation of 
nanoparticles". Dr Yuichiro Kudo (Kitasato University, School of Medicine, Japan) then presented results pertaining to "The evaluation of the safety of asbestos substitutes". Professor Akira Ogami (Institute of Industrial Ecological Sciences, University of Occupational and Environmental Health, Japan) then focused on "Inhalation and instillation studies of submicron and nano particlesimportance of characterization of particles". Finally, Professor Otsuki delivered a summarized presentation on
"Cytokine alteration and speculated immunological pathophysiology in silicosis and asbestos-related diseases".

In this special feature of Environmental Health and Preventive Medicine, Dr Hirano, Dr Kudo and Prof. Otsuki provide a brief review of their specialties. All members of the JSH, irrespective of their attendance at the abovementioned symposium, can enjoy this special feature and obtain detailed information concerning recent advances in studies of fibrous and particulate substances. 